\title{
Vibroacoustics and wave propagation of novel chiral honeycombs
}

\author{
Kong Fah Tee ${ }^{1}$, Alessandro Spadoni ${ }^{2}$, Fabrizio Scarpa ${ }^{1}$ and Massimo Ruzzene ${ }^{2}$ \\ ${ }^{1}$ Department of Aerospace Engineering, University of Bristol, UK \\ ${ }^{2}$ School of Aerospace Engineering, Georgia Institute of Technology, Ferst Drive, GA
}

\begin{abstract}
Novel tetrachiral honeycomb structures are evaluated for the first time from the vibroacoustic point of view. A numerical method based on Bloch wave approximations for Finite Element models of the unit cells is applied to simulate the pass-stop band characteristics of these cellular solids. Experimental modal analysis and modal densities are measured on honeycomb panels and sandwich plate, and the results evaluated with the experimental findings. The novel tetrachiral honeycombs show pass-stop band characteristics with isotropic acoustic signature, while sandwich structures made with the same honeycomb cores have the interesting feature of presenting a high-pass frequency behavior on the same pass-stop bands of the honeycomb.
\end{abstract}

Keywords: Wave propagation, modal density, chiral honeycombs, negative Poisson's ratio

\section{Introduction}

Chiral honeycombs are a subset of cellular materials featuring in-plane negative Poisson's ratio (NPR) behavior. NPR is admissible within the classical linear elastic theory, with a range of the Poisson's ratio for unconstrained, isotropic, 3D solids between -1 and 0.5 . While 0.5 corresponds to incompressible rubber-like materials, the value of -1 would lead to an infinite shear modulus. If the solid is constrained during loading, the equivalent Poisson's ratio coefficient $v$ would be $<-1(-\infty<v<-1)$, or $>0.5$. For special orthotropic materials, such as centresymmetric honeycombs with hexagonal configurations, the Poisson's ratios $v_{12}$ and $v_{21}$ could attain even higher values, having only to satisfy the cross-product relation between Young's moduli and Poisson's ratios $E_{1} v_{21}=E_{2} v_{12}$. [1] Centresymmetric NPR honeycombs have shown increased out-of-plane compressive strength and transverse shear, especially for thin gauge thickness [2]. More importantly, the NPR behavior leads to the sinclastic curvature feature - a NPR panel when bent, assumes a dome-shaped structure, with low in-plane buckling and enhanced manufacturing for complex shape structures. However, centresymmetric configurations are inherently anisotropic, a characteristics which is not always welcome by sandwich structure designers. On the opposite, chiral honeycombs are isotropic structures, with an in-plane Poisson's ratio of -1 . A typical chiral configuration would have unit cells composed by a central cylinder, with tangent ligaments connecting cylinders from neighboring cells. When subjected to in-plane loading, the cylinders would rotate, leading to winding/unwinding of the ligaments, and therefore providing the NPR effect. The Poisson's ratio value of -1, matching the one of the elasticity limit, makes chiral structures extremely interesting, due to their enhanced in-plane torsional stiffness and deformation characteristics, for applications not only limited to classical sandwich structures, but also wing box sections with morphing characteristics $[3,4,5]$. The first chiral topology was proposed at molecular level by Wojciechowski [6], and as structural honeycomb component by Prall and Lakes [7]. More recently, chiral honeycombs have been evaluated for their compressive strength [9], and wave propagation characteristics [5]. The chiral configurations so far considered in open literature are the hexachiral ones - each cylinder connected by 6 tangent ligaments. However, more recently different chiral tessellation has been proposed, almost all leading to in-plane NPR. A specific chiral topology is the tetrachiral one, where the cylinder is connected by 4 tangent ligaments. The peculiarity of this chiral morphology is the fact that it can be tessellated in such a way to obtain a centresymmetric configuration - the whole honeycomb would be constructed simply by translation of the unit cell in the various directions, rather than combined rotations and translations along the tangent directions only [8].

Honeycomb structures, being used in sandwich applications for aerospace and marine environments, are particularly subjected to vibroacoustic analysis to reduce the acoustic signature of the structural components. A vibroacoustic assessment on hexachiral structures has been presented in a previous SPIE Conference [10]. The Authors have also in the past examined the wave propagation behavior of NPR centresymmetric honeycombs making use of a Bloch wave

Active and Passive Smart Structures and Integrated Systems 2008, edited by Mehdi Ahmadian Proc. of SPIE Vol. 6928, 69280J, (2008) -0277-786X/08/\$18 - doi: 10.1117/12.776075 
approach to use Finite Element models of unit cells to calculate pass-stop band characteristics of centresymmetric hexagonal honeycombs showing NPR characteristics. For these honeycombs configurations, strong wave directionality was observed, due also to the anisotropy of the materials [11]. In this work we present an assessment on novel tetrachiral structures from a wave propagation and vibroacoustic point of view, especially to assess if the classical numerical and experimental tools for commercial sandwich constructions could be used also for these structures with non-affine deformation characteristics. We determining the pass-stop band characteristics through the calculations of phase constant surfaces, and experimentally identifying modal properties and modal densities of these cellular structures. The test cases were a honeycomb panel, and a sandwich plate made of quasi-isotropic face skins and tetrachiral RP core. The results show that the honeycomb behaves in a isotropic fashion in terms of dependency of wave vector components, with well separated pass-stop band surfaces. When sandwiched between face skins, however, the honeycomb feature a large stop-band, leaving the other phase constant surfaces almost unchanged. This type of behavior is reflected by the modal density distribution of the structures. From a numerical design point of view, the pass-stop band characteristics can easily be tuned, due to the sensitivity of the phase constant surfaces to the geometric parameters of the unit cells.

\section{Modeling}

The propagation of flexural waves in the tetrachiral structures has been performed using Bloch wave approaches for harmonic propagating waves [12]. The governing equations for a homogenous anisotropic body with linear elastic properties are:
$\sigma_{i j, j}=\rho \frac{\partial^{2} u_{i}}{\partial t^{2}}$

$$
\sigma_{i j, j}=C_{i j k l} \varepsilon_{k l}
$$$$
\varepsilon_{k l}=\frac{1}{2}\left(u_{k, l}+u_{l, k}\right)
$$

The solution for generalized displacements is the following:

$u_{m}\left(x_{n}, t\right)=U_{m}\left(x_{n}\right) e^{-i \omega t}$

Using (2), the stress and strain tensors of the body become complex functions of the type:

$$
\varepsilon_{i j}\left(x_{n}, t\right)=\mathrm{E}_{i j}\left(x_{n}\right) e^{-i \omega t} \quad \sigma_{i j}\left(x_{n}, t\right)=\Sigma_{i j}\left(x_{n}\right) e^{-i \omega t}
$$

Substituing (2) and (3a, 3b) in (1a, 1b, 1c), the new governing equations of motion become:

$$
\Sigma_{i j, j}+\rho \omega^{2} U_{i}=0 \quad \Sigma_{i j}=C_{i j k l} E_{k l} \quad E_{k l}=\frac{1}{2}\left(U_{k, l}+U_{l, k}\right)
$$

Using Bloch's theorem one can express the complex generalized displacements on a periodic unit cell (Figure 1a), as:

$U_{m}\left(x_{n}, t\right)=U_{m}\left(x_{n}+l_{n}\right) e^{-i k n_{j} l_{j}}$

The degrees of freedom (DOFs) of a centresymmetric unit cell can be decomposed into left (L), right (R), top (T) and bottom (B). The Floquet conditions for wave propagation in a Cartesian $x y$ coordinates frame are the following:

$$
U_{B}=U_{T} e^{-i k_{y} y} \quad U_{L}=U_{R} e^{-i k_{x} x}
$$

Because of the complex terms, relation (5) can be decomposed as:

$$
U_{m}\left(x_{n}\right)=U_{m}^{\mathrm{Re}}\left(x_{n}\right)+i U_{m}^{\operatorname{Im}}\left(x_{n}\right)
$$


Imposing (7) in (1b), the new equations of motion become:

$\sum_{i j, j}^{\mathrm{Re}}+\rho \omega^{2} U_{i}^{\mathrm{Re}}=0 \quad \sum_{i j, j}^{\mathrm{Im}}+\rho \omega^{2} U_{i}^{\mathrm{Im}}=0$

The implementation of $(8 \mathrm{a}, 8 \mathrm{~b})$ in a Finite Element implicit method lead to the creation of two superimposed meshes, one corresponding to the real part, the other to the imaginary part. The connection between the boundary DOFs of the two meshes is performed using the following periodic BCs:

$U_{1}^{\mathrm{Re}}=U_{2}^{\mathrm{Re}} \cos \left(k n_{j} l_{j}\right)+U_{2}^{\mathrm{Im}} \sin \left(k n_{j} l_{j}\right) \quad U_{1}^{\mathrm{Im}}=U_{2}^{\mathrm{Im}} \cos \left(k n_{j} l_{j}\right)-U_{2}^{\mathrm{Re}} \sin \left(k n_{j} l_{j}\right)$

Where $k_{n} j j$ are the propagation constants of the wave vector. Considering the first Brillouin zone $-\pi \leq k_{x}, k_{y} \leq \pi$, the BCs $(9 \mathrm{a}, 9 \mathrm{~b})$ can be rewritten in terms of the $x$ and $y$ components of the wave vector:

$U_{\text {bottom }}^{\mathrm{Re}}=U_{\text {top }}^{\mathrm{Re}} \cos \left(k_{y}\right)+U_{\text {top }}^{\mathrm{Im}} \sin \left(k_{y}\right)$

$U_{\text {left }}^{\mathrm{Im}}=U_{\text {right }}^{\mathrm{Im}} \cos \left(k_{x}\right)-U_{\text {right }}^{\mathrm{Re}} \sin \left(k_{x}\right)$

$U_{\text {left }}^{\mathrm{Re}}=U_{\text {right }}^{\mathrm{Re}} \cos \left(k_{x}\right)+U_{\text {right }}^{\mathrm{Im}} \sin \left(k_{x}\right)$

$U_{\text {bottom }}^{\mathrm{Im}}=U_{\text {top }}^{\mathrm{Im}} \cos \left(k_{y}\right)-U_{\text {top }}^{\mathrm{Re}} \sin \left(k_{y}\right)$

The finite element models can therefore be coupled using the relations (10-13). For a given set of $k_{x}$ and $k_{y}$, the eigenvalues of $(8 \mathrm{a}, 8 \mathrm{~b})$ can be calculated using a Block Lanczos algorithm. The solutions of the eigenvalue problem constitute the phase constant surfaces of the periodic structure, providing information on the stop-pass bands of the honeycomb. The total number of modes $N$ less than a specified frequency $\omega_{0}$ for a honeycomb panel made of $N_{1} N_{2}$ cells in the $x$ and $y$ directions can be derived from the following expression [13]:

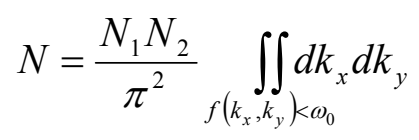

Where $f\left(k_{x}, k_{y}\right)$ is a phase constant surface. The modal density for a single phase constant surface can be estimated numerically as:

$$
n=\frac{\partial N}{\partial \omega_{0}}
$$

The modal density for the whole honeycomb structure can be evaluated calculating the number of modes (14) for each phase constant surface, summing all the contributions from the different $f\left(k_{x}, k_{y}\right)$, and calculating the gradient of the sum versus the frequency as per (15). 




Figure 1. Periodic unit cell of tetrachiral honeycomb

\section{Manufacturing and testing}

Honeycomb tetrachiral structures were manufactured using a Rapid Prototyping SLS technique oout of HP PA synthered powders. The aspect ratio $\alpha$ of the cells (length of straight side/radius of cylinder) was 4.4 , with a relative thickness $\beta$ (thickness of straight side/radius of cylinder) equal to 0.15 . The length of the straight side was $2.5 \mathrm{~cm}$, with a gauge thickness (height) of $5 \mathrm{~cm}$. The total honeycomb panel room was $22.5 \mathrm{~cm} \mathrm{X} 20 \mathrm{~cm} \mathrm{X} 5 \mathrm{~cm}$. The Young's modulus of the core material measured on a BSI standard sample under tensile loading was 1.6 GPa. A sandwich panel was build using a similar core with 2 face quasi-isotropic composite face skins made with a 8552 prepreg with a $0 /+45 /-45 / 90 / 90 /-45 / 45 / 0$ stacking layer sequence. The face skins and the core were attached with a epoxy-resin based adhesive and cured in autoclave at $120^{\circ}$.

One tetrachiral honeycomb core panel and one analogous sandwich panel (Figures 1a and b) were tested using a scanning laser vibrometer (Polytec PSV-300 F). An electrodynamic shaker (Ling Dynamic Systems V406)) was used to provide white noise broadband base excitation. A D_Space-based signal generator provided input to the shaker through a power amplifier (model LDS PA100E). Input force was detected using a PCB 208C03 force transducer. The resolution, force range, sensitivity and frequency range of force sensor are $0.005 \mathrm{lb}(0.022 \mathrm{~N}) \mathrm{rms}, \pm 500 \mathrm{lb}(2224 \mathrm{~N})$, $2.37 \mathrm{mV} / \mathrm{N}$ and $36 \mathrm{kHz}$, respectively

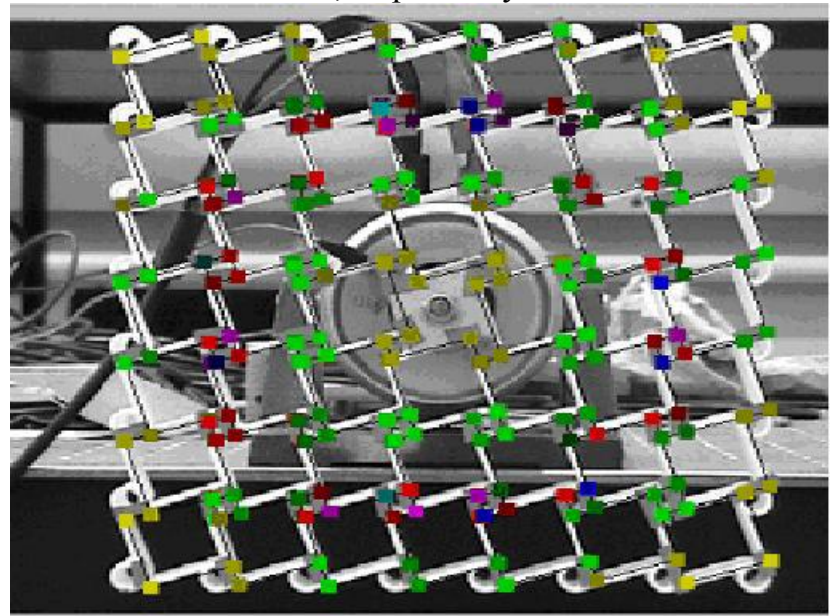

Figure 2. Core panel

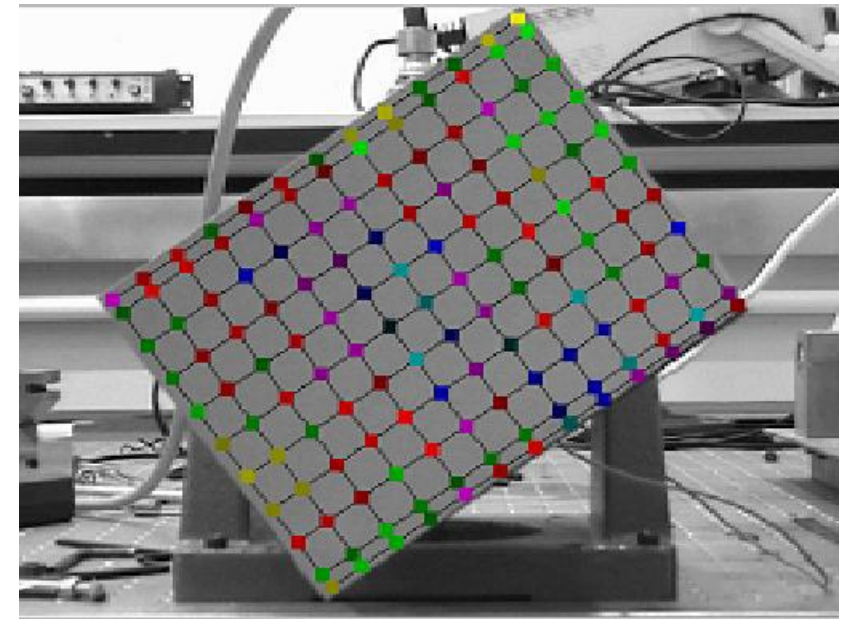

Figure 3. Sandwich Panel

The tetrachiral honeycomb core panel was connected to the shaker using a stinger and two thin aluminium square base plates attached to the center. For the tetrachiral sandwich panel, the force sensor was connected to the panel using thin aluminum round base plate fixed on the center (Figure 3).

The velocity range for the SLV corresponding to a vibrometer output voltage of $1 \mathrm{~V}$ was $1 \mathrm{~mm} / \mathrm{s}$. A FFT acquisition was performed within a selected bandwidth between 0 and $5 \mathrm{kHz}$. The SLV was used to perform a modal analysis of the 
tetrachiral honeycomb and sandwich panel, as well as the measurements of the modal densities of the cellular structures. For the modal analysis, the input was a random excitation, whereas 252 points on the surface of the tetrachiral honeycomb core panel were used as measurement locations, each point scanning and FFT averaged 20 times. For the tetrachiral sandwich panel, 143 points were scanned. FRFs for each data point and average FRF were obtained, and in a universal file (.unv) for post-processing using the LMS modal analysis software. The experimental modal densities have been derived from the averaged point mobility $Y$ through the expression [14]:

$$
n(f)=4 m<\operatorname{Re}(Y)>
$$

Where $m$ is the mass of the panel and $<\operatorname{Re}(Y)>$ is the spatial average of the real part of the driving point admittance. Driving point admittance, $Y$ is the ratio of the Fourier transform of the velocity of the excitation point to the Fourier transform of the excitation force, being equivalent to a FRF, which can be obtained directly from the scanning laser vibrometer. Nine different excitation point locations were used for the modal density of the tetrachiral honeycomb core panel, with the real part of the driving point admittances averaged spatially using the 2-way channel technique. For the tetrachiral sandwich panel, one center excitation point was used instead.

\section{Results}

\subsection{Numerical modeling}

The first four phase constant surfaces for both the tetrachiral honeycomb core and sandwich unit cells are shown in Figs. 4 and 5, respectively corresponding to different pass-stop bands. The phase constant surfaces are plotted with both $\varepsilon_{1}$ and $\varepsilon_{2}$ varying in the range from $-\pi$ to 0 for symmetry reasons.

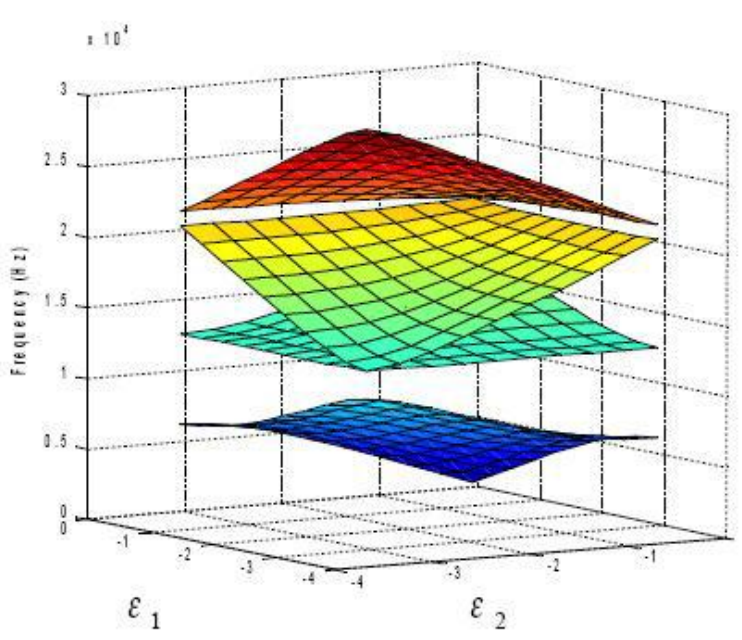

Figure 4

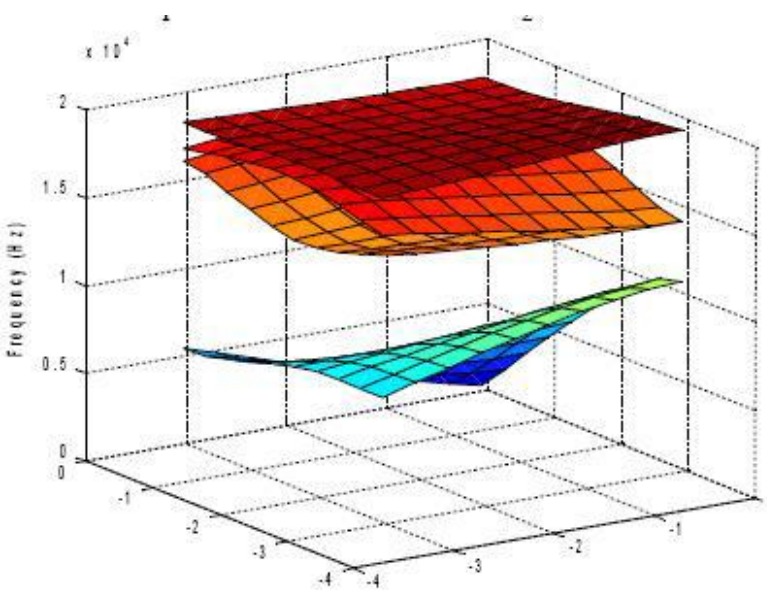

Figure 5

It is interesting to notice how the presence of the face skins on the honeycomb unit cells provides a shift between the first and second pass-stop band of $1.5 \mathrm{kHz}$ on average, at the same time slightly lowering down the last 3 phase constant surfaces. In terms of behavior versus the propagation constants, the presence of the face skins still maintain a minimum for $(0,0)$, while the maximum is now moved to $(0,-\pi)$ for the sandwich construction. The $3^{\text {rd }}$ phase constant surface area is also significantly reduced for the sandwich case. An important aspect when considering the wave propagation behavior in honeycomb cores is the SH-type of shear wave one. Wave modes assumed by the unit cell depend on the Poynting vector, i.e., the wavenumber components. Figures 6 shows the first 3 modes associated to $(-\pi, 0)$, and geometry parameters $\alpha=4, \beta=0.1$, for gauge thickness equal to unity. The modes provide different out-of-plane bending effects, where the cylinder start to deform significantly only at the third surface constant. For a wave propagating at $45(-\pi,-\pi)$ with the same geometric and material properties characteristics, the $1^{\text {st }}$ mode provides out-of-plane deformations in antiphase compared to the previous case at 0 , while there appear to be a mode veering between the $2^{\text {nd }}$ and $3^{\text {rd }}$ of the ($\pi, 0)$ and $(-\pi,-\pi)$, where the modeshapes interchange, and the $2^{\text {nd }}$ mode provide the most significant cylinder deformation 
(Figures 7). To estimate the overall vibroacoustic behavior of the honeycomb, it is necessary to take extra care in integrating (14) when surface veering occurs.


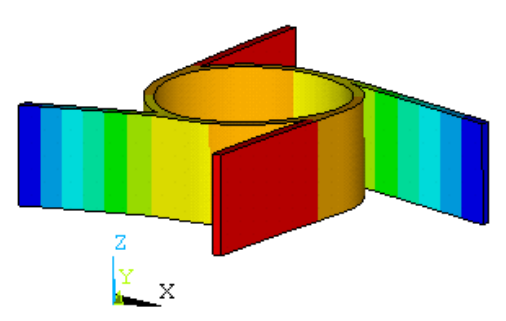

Figure 6a
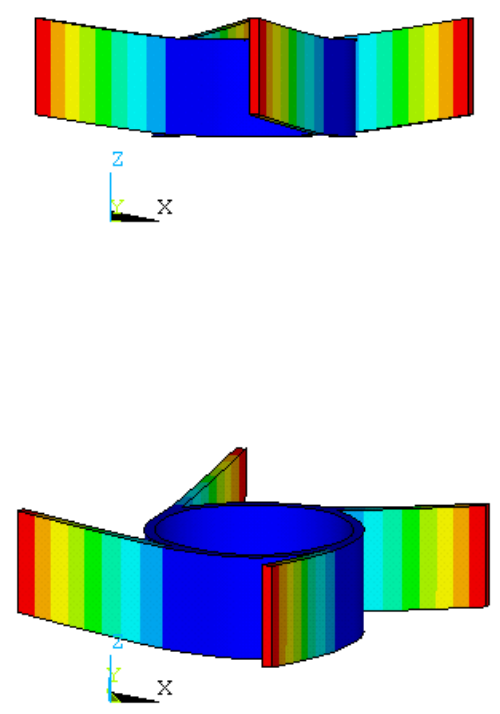

Figure 7a

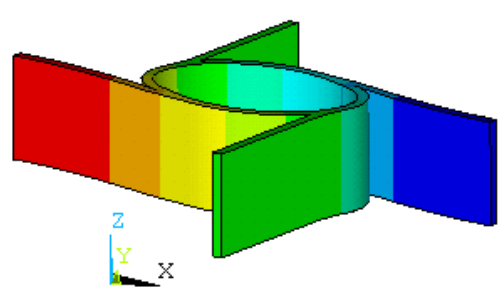

Figure 6b


Figure 7b

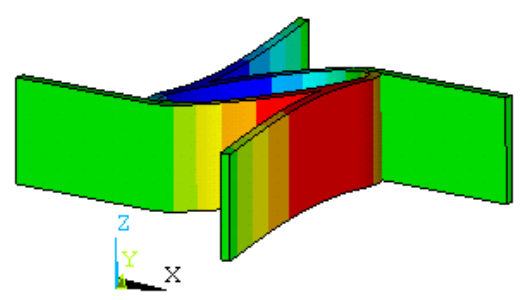

Figure 6c
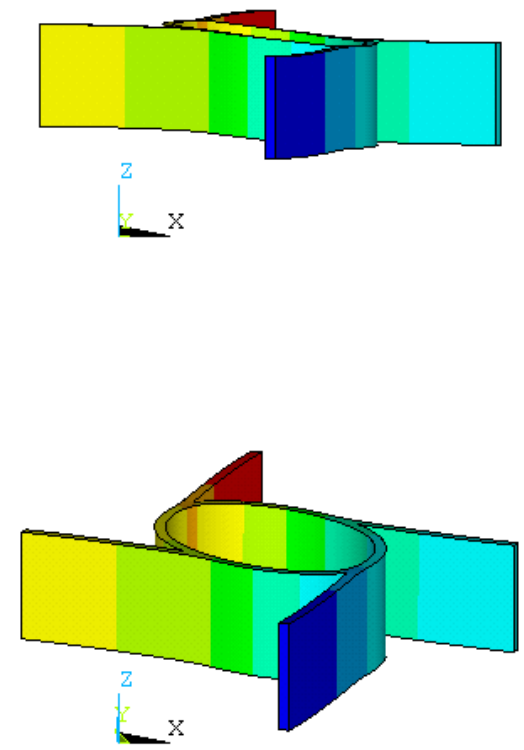

Figure 7c

\subsection{Modal analysis and modal density}

Figure 8 shows the average experimental FRF for both the tetrachiral honeycomb core and sandwich panels. Already from the $\mathrm{H} 1$ average indicator, it is evident that the presence of the face skins contributes to eliminate some modes, while passing different ones, associated with higher transverse shear deformations. The identified experimental natural frequencies and modal loss factors for both the tetrachiral honeycomb core and sandwich panels are presented in Table 1. It is worth noticing that the first mode calculated from the FE model for $(0,0)$ provides a vakue of $380 \mathrm{~Hz}$, while the first experimental natural frequency is $309 \mathrm{~Hz}$. The experimental setup involved the presence of two aluminum bases 
constraining the central point of the honeycomb, therefore providing an added artificial BC condition, and possibly lowering the effective value of the first bending mode for the chiral panel. The first natural frequency of the sandwich panel is also significantly damped, due to the high constraining shearing effect given by the core, bending of the face skins and the adhesive. The modal loss factors associated to the honeycomb panel alone are quite low, as expected due to the dissipative characteristics of the core material alone.

It is interesting also to note the synclastic curvature effect that is present in the global mode shapes of the tetrachiral honeycomb (Fig. 9 and 10). The first bending mode (1,1) (Figure 9) features the double curvature characteristics, which are also present in the $2^{\text {nd }}$ one $(2,1)$. This is a further indication that the tetrachiral structure behaves globally like an isotropic homogenous material with Poisson's ratio -1.

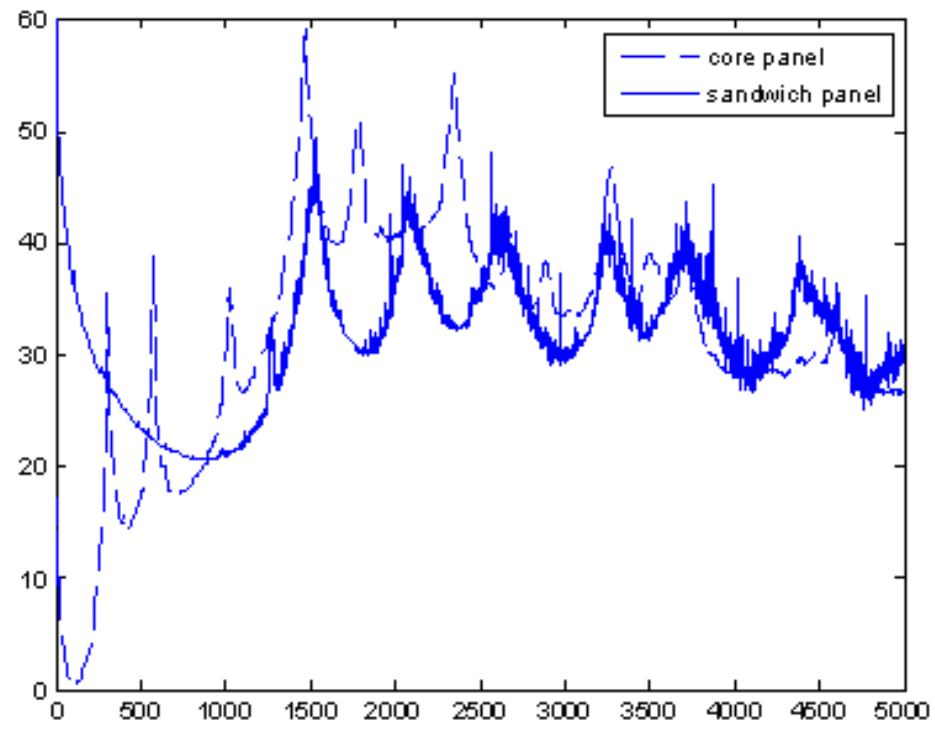

Figure 8.

\begin{tabular}{|c|c|c|c|c|}
\hline \multirow{2}{*}{ Mode } & \multicolumn{2}{|c|}{ Tetrachiral Core Panel } & \multicolumn{2}{c|}{ Tetrachiral Sandwich Panel } \\
\cline { 2 - 5 } & $\begin{array}{c}\text { Natural } \\
\text { Frequencies (Hz) }\end{array}$ & $\begin{array}{c}\text { Modal Loss Factor } \\
(\%)\end{array}$ & $\begin{array}{c}\text { Natural } \\
\text { Frequencies (Hz) }\end{array}$ & $\begin{array}{c}\text { Modal Loss Factor } \\
(\%)\end{array}$ \\
\hline 1 & 308 & 1.7 & 784 & 8.0 \\
\hline 2 & 570 & 0.7 & 1272 & 0.4 \\
\hline 3 & 1019 & 0.8 & 1522 & 2.4 \\
\hline 4 & 1467 & 0.8 & 2089 & 3.1 \\
\hline 5 & 1786 & 0.8 & 2597 & 3.0 \\
\hline 6 & 2347 & 0.8 & 3269 & 1.8 \\
\hline 7 & 2695 & 1.1 & 3655 & 1.2 \\
\hline 8 & 2886 & 1.0 & 4389 & \\
\hline 9 & 3275 & 0.9 & & \\
\hline 10 & 3506 & 1.0 & & \\
\hline 11 & 3722 & 1.2 & & \\
\hline
\end{tabular}

Table 1 


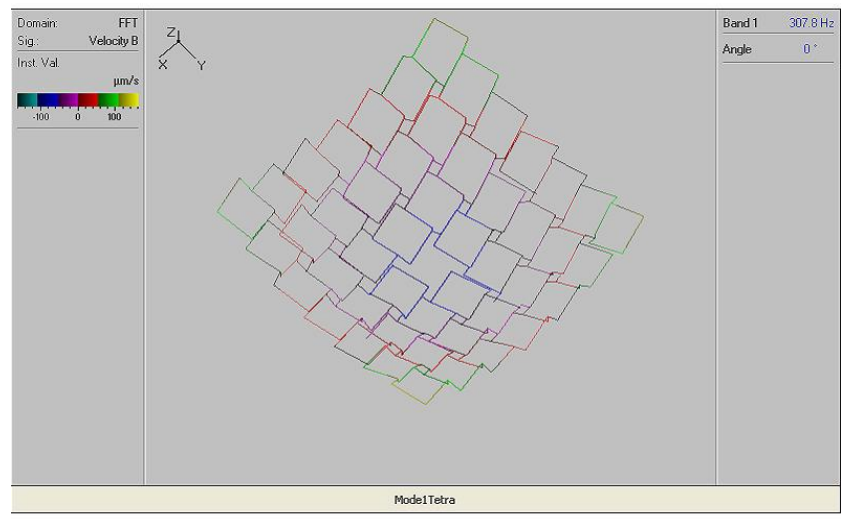

Figure 9

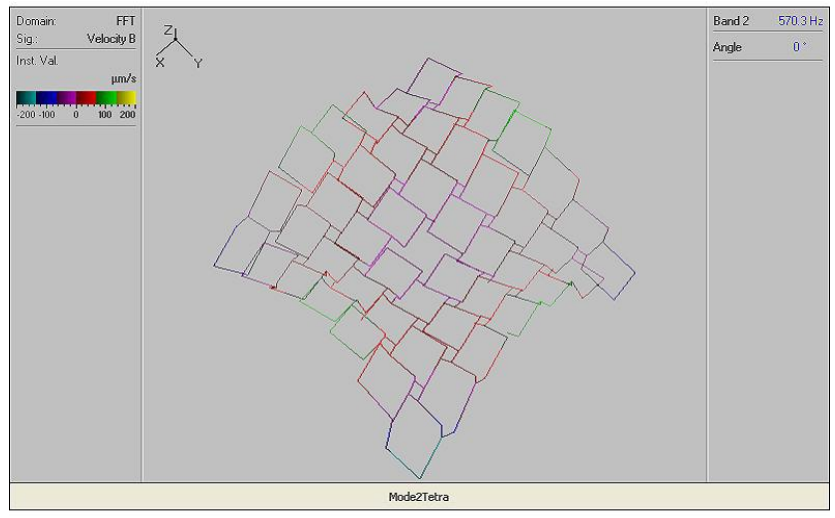

Figure 10

The experimental modal densities of the two panels, as given by Eq. (16), are shown in Fig. 10. Similarly to the average H1 indicator, the averaged point mobility shows that the presence of the face skins act as high-pass filter for phase constant surfaces higher than the third of the honeycomb panel, and only highly coupled shearing-bending modes give provide contributions to significant resonant behavior.

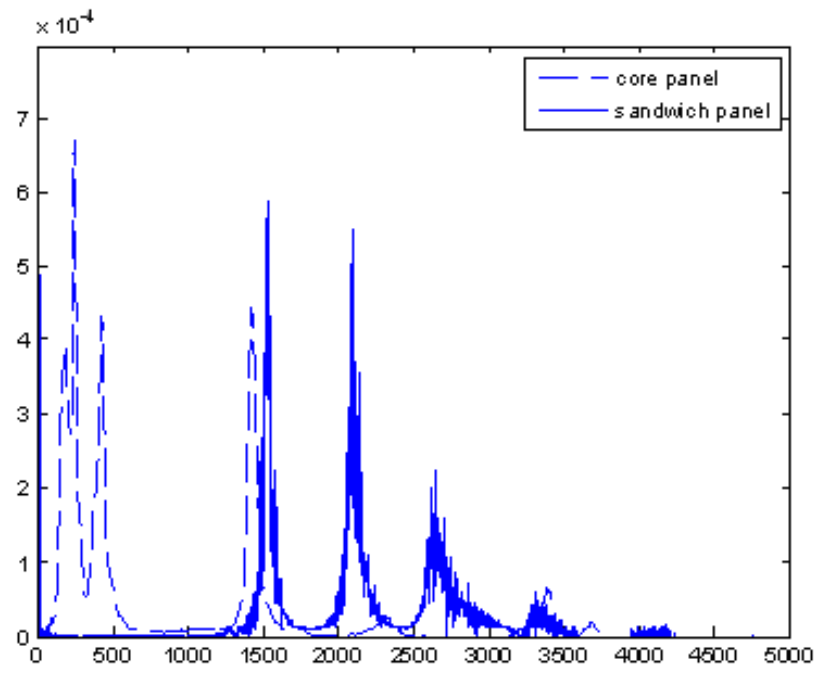

\section{Figure 11}

An important parameter for the selection of honeycomb materials is the relative density, not only for the compressive and strength properties, but also for the stiffness to weigh ratio. Chiral honeycombs perform interestingly, from that point view [10]. Figure 12 shows the thickness ratio required to have the same density of a commercial honeycomb, with scalable cell wall aspect ratio and relative density. A chiral honeycomb could be manufactured with a lower aspect ratio $\alpha$, and lower straight side thickness (4 times lower) compared to a hexagonal honeycomb, to have the same overall mass density. This feature could be beneficial for room size, and minimization of wasted material during processes like Resin Transfer Moulding. 

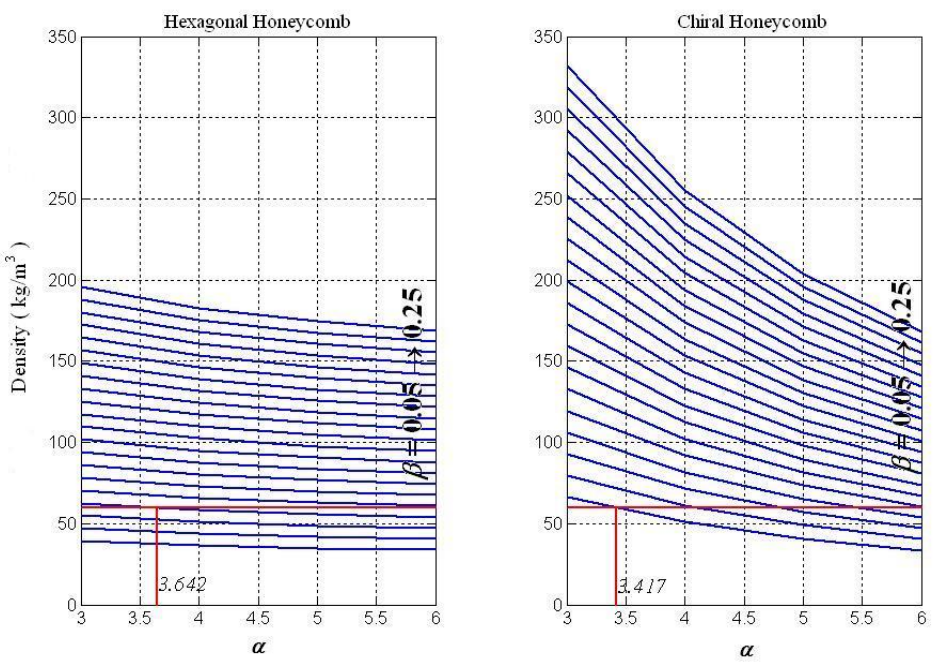

Figure 12

\section{Conclusions}

This initial study has shown that novel tetrachiral honeycomb structures can be modeled using tools for classical and commercially available sandwich structures. Moreover, the tetrachiral honeycomb shows an isotropic acoustic signature at lower modes. Tetrachiral honeycombs have also isotropic mechanical properties, coupled with synclastic curvature features that make them very attractive to manufacture curved or complex shape sandwich components. This characteristics, coupled with their vibroacoustic behavior and enhanced compressive strength of chiral honeycombs compared to commercial hexagonal ones with the same low relative density, suggest potential benefits on adopting the tetrachiral topology for future applications.

\section{Acknowledgements}

This work has been partially funded through the FP6-NMP-2005-STRP-01364 Programme CHISMACOMB

\section{References}

1. Rod Lakes, K. W. Wojciechowski. Physica Status Soldi B, 245(3), 545-551 (2008)

2. F. Scarpa, G. Burriesci, F. C. Smith and B. Chambers. The Aeronautical Journal, March 2003, pp 175-183 (2003)

3. Jamie Martin, Jean-Jacques Heyder-Bruckner, Chrystel Remillat, Fabrizio Scarpa, Kevin Potter, Massimo Ruzzene. Physica Status Solidi B, 245(3), 570-577 (2008).

4. D. Bornengo, F. Scarpa, and C. Remillat, Proc. IMechE - Part G, J. Aerospace Eng. 219, 185-191 (2005)

5. A. Spadoni, M. Ruzzene, and F. Scarpa, J. Intell. Mater. Syst. Struct. 17(11), 941-952 (2006)

6. K. W. Wojciechowski, Phys. Lett. A 137, 60-64 (1989).

7. D. Prall and R. Lakes, Int. J. Mech. Sci. 39, 305-314 (1996).

8. Joseph N. Grima, Ruben Gatt, Pierre-Sandre Farrugia. Physica Status Solidi B, 245(3), 511-520 (2008)

9. F. Scarpa, S. Blain, T. Lew, D. Perrott, M. Ruzzene and J.R. Yates. Composites Part A, 38(2), 280-289 (2007).

10. Tze Ling Lew, Alessandro Spadoni, Fabrizio Scarpa, Massimo Ruzzene. Chiral hexagonal cellular sandwich structure: a vibro-acoustic assessment. Proceedings of SPIE -- Volume 5760, Smart Structures and Materials 2005: Damping and Isolation, Kon-Well Wang, Editor, May 2005, pp. 559-568

11. Ruzzene M., Soranna F., Scarpa F. Smart Materials and Structures, 12, 363-372 (2003).

12. M. Aberg and P. Gudmundson. J. Acoust. Soc. Am. 102(4), 2007-2013 (1997)

13. R S Langley. J. Sound Vib. 172(4), 491-511 (1994).

14. K Renji. J. Sound Vib. 237(1), 67-69 (2000) 